\section{Translational Cardiology: defining limits and targets at the International Archives of Medicine}

\section{Translational research as a tópos}

The Merriam-Webster dictionary reports the first use of "Translational Research" as early as 1986 and the definition given for this term was: "medical research that is concerned with facilitating the practical application of scientific discoveries to the development and implementation of new ways to prevent, diagnose, and treat disease, called also translational medicine" [1]. The adjective "translational" stems from Classical Latin "translatio", then probably Middle French "translation", to reach Middle English "translacioun". The idea is to make a transfer from one idiom to another, yet keeping the intimate significance and sense, which is common to language translation methods. It properly stresses the relation to the transfer of scientific knowledge into practical applications. It is obvious how, in less than 30 years, translational research has pervaded all Science domains and how large is the interest of scientists and lay people for this subject.

Medicine in general was particularly seduced by the translational concept, probably for its peculiar applied scopes. Cardiology was not an exception. Just to quote few among several examples: a) the International Society for Cardiovascular Translational Research [www.isctr.org] was formed in 2007 with the mission to provide an environment for collaboration and guidance among basic and clinical scientists, regulatory authorities and the medical industry to expedite scientific discoveries into clinical applications, disseminate the science among the scientific community and promote research, development of guidelines for training and certification in Translational Research, influence health care policy, educate the public, and improve the well being of patients. Regular symposia have now reached the $7^{\text {th }}$ edition and the Society is expanding; b) a Journal of Cardiovascular Translational Research [ISSN: 1937-5387 (Print); 1937-5395 (Online)] was published by Springer starting 2008; as of 2013 it has a fair Impact Factor of 2.691 with regular issues every other month,

\section{Paolo Emilio Puddu} MD, PhD', Cesare MN Terracciano $\mathrm{MD}, \mathrm{PhD}^{2}$

1 Department of Cardiovascular Sciences, Sapienza, University of Rome, Rome, Italy.

2 National Heart \& Lung Institute, Imperial College London, London, United Kingdom.

Contact information:

Paolo Emilio Puddu.

$\risingdotseq$ paoloemilio.puddu@uniroma1.it

Cesare MN Terracciano.

झ c.terracciano@imperial.ac.uk 
monthly from October 2014; c) the newly selected Editor-in-Chief of the Journal of the American College of Cardiology has implemented a special, blue highlighted, Perspectives window on published articles, whereby the Translational Outlook is given, which stresses the importance of what is presented and why it might deserve further applicability. For example, Martínez-Legazpi et al. concluded that using the intraventricular vortex ring, the normal ventricle in sinus rhythm enters $10 \%$ to $15 \%$ of its filling volume at no metabolic or pressure cost; consequently enhanced vortex function reduces chamber operative stiffness in conditions of eccentrically remodeled ventricles, such as non ischemic dilated cardiomyopathy whereas reduction of vortex-mediated filling is a mechanism of diastolic dysfunction in severe concentric remodeling diseases, such as hypertrophic cardiomyopathy [2]. The Journal's translational outlook was that "Impaired vortex flow can be used to evaluate interventions and devices that influence diastolic intraventricular flow and global diastolic function in patients with various forms of acute and chronic cardiac disease." [2].

The director of the National Institute of Neurologic Diseases and Stroke (NINDS) posted on March 2014 a blog on secular changes in the nature of research supported by the Institute. Noting that official definitions of basic and applied research were not helpful, the NINDS defined basic research as "an understanding of the structure and function of the nervous system," whereas applied research was "aimed at developing and testing diagnostics, therapeutic agents, or preventive interventions." They subclassified basic research into "basic/basic," which "focused on understanding the normal nervous system," and "basic/disease-focused," which "focused on understanding disease mechanisms." The NINDS found a marked decline, from 1997 to 2012 , in support for basic research (from $87 \%$ to $71 \%$ of expenditures), accompanied by a marked increase (from $13 \%$ to $29 \%$ ) in applied research. What the NINDS subclassified as basic/basic research had the steepest decline in applications and financial support (from 52\% to 27\%): they commented that possibly the applicants themselves might have just thought that the NINDS had simply low interest in that type of research. Recognizing the need to play a bigger role in bridging the gap between the discovery of a potential therapy and its development and clinical testing, the NINDS has just launched (August $13,2014) 3$ carefully crafted funding programs, each tailored to specific treatment modalities and 2 of these, CREATE (Cooperative Research to Enable and Advance Translational Enterprises) Bio, and CREATE Devices are fundamentally Translational Research programs to offer support for preclinical development and potentially small clinical trials and allow researchers in academia and small companies the opportunity to play a more active part in translating their basic neuroscience discoveries into treatments [3].

In the launching Editorial of the new Elsevier Journal EBioMedicine in November 2014 "as a centralized forum where clinicians and scientists in both the clinic and the laboratory can communicate their observations, ideas, and research insights and work together to address outbreaks such as Ebola and other major biomedical health challenges" Stacey and Le stated [4] that the new Journal is certainly translational but asked "how we define translational research?" They pointed out that: "Technology has become so powerful and so nimble that personalized whole-genome sequencing is likely to be within our reach as a practical clinical tool. For example, clinicians may now use genomic screening to stratify cancer patients into groups that might benefit from targeted, genespecific treatments that are in turn derived from recent basic science advances in the underlying biology of those altered genes. Translational medicine endeavors to bring basic researchers and clinicians together in a common space where great discoveries in the laboratory can be applied directly 
and rapidly in the clinic. The field also depends on the input and feedback from those at the forefront of clinical care, their daily observations in the clinic being critical for both detecting the emergence of new public health concerns and communicating the progress of new treatment modalities.". They nicely concluded that "Translational research is therefore inherently bidirectional and far reaching in its scope and applications.".

Therefore, in some sparse way, the what translational means in Cardiology, whether it deserves attention, how might it be fostered by Scientific Societies or Public Grant Authorities has been addressed in recent interventions, all sharing the need of stimulating a closer relation between basic scientists (from biology $[3,4]$ to epidemiology [5]) and applied clinicians, also bidirectionally [4]. It is less clear for whom and why. When a short Perspective paragraph is put in comment and highlight of a relatively complex and quite long manuscript [2] it is not always easy for a Reader to completely understand/translate the message and then apply the presented new evidence or concept to the daily care, finding the full significance of the translational concept. The reverse should also be true: how could the over-occupied clinician devote time and attention to a conversation with a fundamentalist in order to ask questions that may be replied from the bench? It is therefore needed, as in beautiful translations for Literature or Poetry of different cul- tures into our own, that experts in the two languages (and cultures) act as translators and in order to be effective some rules should be followed not limited to word by word transfer but entering sense, tune and philosophy.

Translational research might be a Tópos ( $\tau$ ó $\pi$ os, in Greek 'place' from tópos koinós, common place), in Latin locus (from locus communis), referred in the context of classical Greek rhetoric to a standardised method of constructing or treating an argument ${ }^{1}$. Tópoi (the plural) can be used to invent arguments and also to conceptualize and formulate the singlesentence declarative thesis. They were defined as "ways of probing one's subject in order to find the means to develop that subject" [6]. There are 4 Tópoi that are most useful to students and/or scholars: definition, analogy, consequence, and testimony ${ }^{2}$. Teaching the Tópoi requires using examples and good examples are to be had by applying each topic to a definite subject and coming up with several thesis statements [6].

It is the purpose of the present Editorial to offer the Readership of the International Archives of Medicine the rationale of 2 most important Tópoi. Thus we explicitely address the "why" and the "to whom" we decided that a Translational Cardiology Section was appropriate to fill the gap between basic and clinical Science. To be pragmatic few selected examples were chosen and we present the essential of what a manuscript should contain in

1. Aristotle divided Tópoi into "common" and "special" groups. In the common group could be found categories such as laws, witnesses, contracts, oaths, comparisons of similarity, difference, or degree, definitions of things, division of things (whole/parts, for instance), cause and effect, and other items that could be analyzed, researched or documented.

2. Definition involves the creation of a thesis by taking a fact or an idea and explaining it by precisely identifying its nature; it always asks the question "What is/was it?" Analogy is concerned with discovering resemblances or differences between two or more things proceeding from known to unknown; it is a useful tool for investigating comparisons and contrasts because it always asks the question "What is it like or unlike?" Consequence investigates phenomena costs to effect-to-cause pattern, best established through probabilities from patterns that have previously occurred. It always answers the question "What caused/causes/will cause it?" Testimony relies on appeals to an authority (such as an expert opinion, statistics, or the law) and it always answers the question "What does an authority say about it?" "Ultimately a thesis or an argument must say something about the real world." 
order to serve under these flags. Our contribution is allocated under 2 items and it principally offers considerations starting from our own experience, which enabled our critique to be potentially less constrained.

It is almost a requirement for translational research that it should be inspired by the clinic. Translational research is often defined as "bench to bedside" but it is safe to state that the initial step is invariably taken from clinical observations. This is the phase when a particular clinical problem incites the researchers to look into the basic science background and formulate "translational" hypotheses. Therefore, we "reversed" what is considered the "standard" presentation, with the clear-cut allusion to the need of being clinically driven, underpinning what we strongly believe is the force and impact of translational research in Cardiology, namely to be derived from a clinical question and to aim at solving a clinical problem or adding to pathophysiological concepts or mechanisms new elements for prevention, diagnosis or treatment.

\section{Translational research in Cardiology: from the bedside to the bench}

In a recent study using 3 dimensional data obtained by Speckle Tracking Echocardiography (3DSTE) to detect semi-automated and homologous landmarks clouds as proxies of left ventricular heart morphology, an extended Geometric Morphometrics toolkit distinguished between intra- and interindividual shape variations [7]: a special strategy compared the shape, orientation and size of cardiac cycle's morphological trajectories in time. The fundamental, physiologically-based assumption was that shapes of trajectories with inter-individual variation are expressions of left ventricular heart function when compared at electrophysiologically homologous times $[7,8]$. Using a special type of Riemannian Parallel Transport, called "linear shift" human left ventricular heart morphological changes in time were compared among 17 healthy subjects and 2 patients with volumetric overload due to Aortic Regurgitation (AR). Whereas the 2 patients with AR were not differentiated in the static shape analysis from the healthy subjects, they set apart significantly in the analyses of trajectory's shape and orientation. In healthy subjects, the variations due to inter-individual morphological differences were not related to shape and orientation of morphological trajectories. Principal Component Analysis showed that volumetric contraction, torsion and twist are differently distributed on different axes. Moreover, global shape change appeared to be more correlated with endocardial shape change than with the epicardial one. Finally, the total shape variation occurring among different subjects was significantly larger than that observable across properly defined morphological trajectories [7]. This type of investigations was extended, more recently, to patients with hypertrophic cardiomyopathy $(\mathrm{HCM})$ with positive genotyping [9]. Figure 1 illustrates how the analysis of morphological trajectories through time can unveil some pathophysiological differences among heart diseases. A group of healthy subjects $(n=48)$ is compared with a group of Hypertrophic Cardiomyopathy patients ( $\mathrm{HCM}, \mathrm{n}=24$ ) and preliminarily with 2 patients affected by $A R$. This analysis detects significant differences in trajectories orientation in the PC1PC2 shape space. Orientation is defined by the vector connecting PC values at R-peak with those at the end-systolic volume time. The translational example here is represented by the potential to start from a clinically adopted diagnostic tool as 3DSTE whereby morphometric parameters are obtained after dedicated elaborations and then adapt mathematical, statistical and engineering (in case of principal strain lines [8]) tools to finally conclude that volume and pressure overloads have opposite consequences on those parameters. In return these efforts might eventually be used clinically if peculiar 
Figure 1: By defining orientation as the vector connecting Principal Component (PC) values at R-peak with those at the end-systolic volume time, the starting point is common to all studied individuals: a) healthy subjects ( $n=48)$; b) Hypertrophic Cardiomyopathy (HCM, $n=24)$ patients and c) Aortic Regurgitation ( $A R, n=2$ ) patients. Significant differences in trajectories orientation in the PC1-PC2 shape space are seen in HCM versus AR patients. Healthy subjects (whose trajectory orientation line is not significant) stay in the middle.
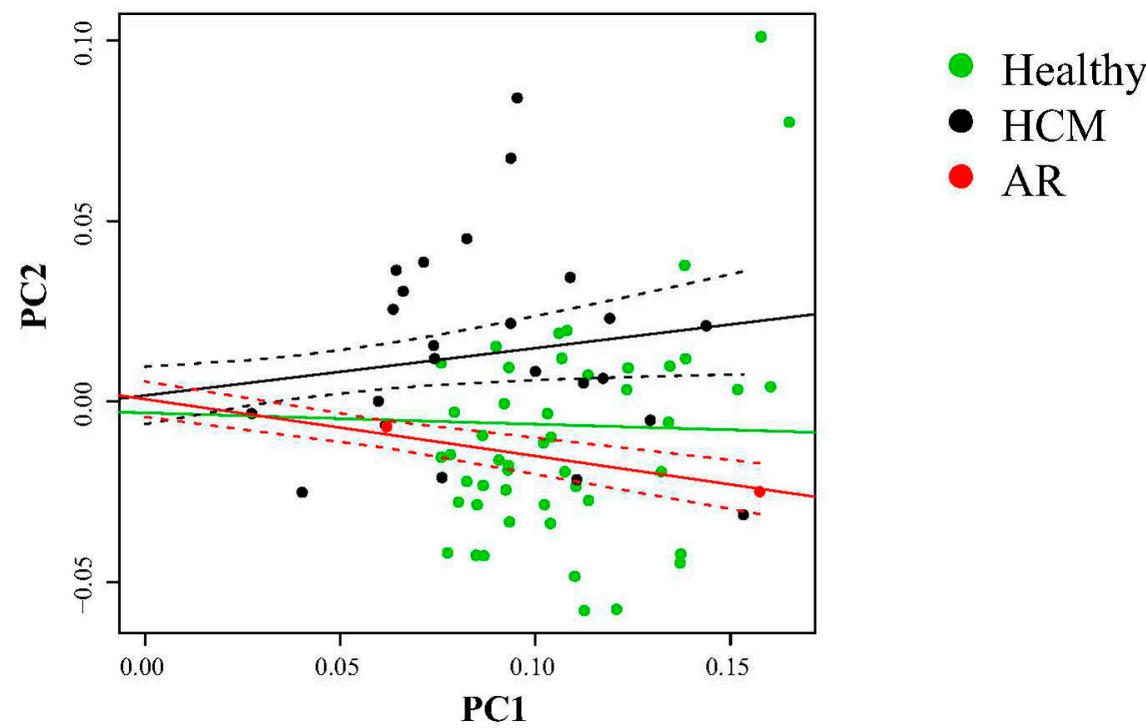

conditions such as genotype positive but phenotype negative HCM individuals might so be disclosed as abnormal [10]. It is interesting that using completely different approaches, the vortex visualized from inside left ventricular wall [2] and morphometrics and principal strain lines evidenced from outside [7-10], both representing medical-engineering interactions, the conclusions are not dissimilar from the translational point of view, namely that impairments represent or index peculiar pathological conditions and may thus be used diagnostically. As these methods are very subtle, it should now be essential to know whether they might be applied early enough to secure diagnostic elements when the situation is not still advanced as is the case by standard methods. Here only should a crown be on such research, which illustrates how important is time in translational research.
In the context of both acute and chronic ischemic heart disease, prolongation of the electrocardiographic (ECG) interval QTC was a predictor of sudden death due to life-threatening arrhythmias as ventricular fibrillation [11-13] which is known from a long time. When standard ECGs were performed in patients intoxicated by glyphosate-based herbicide $(\mathrm{GBH})$ [14] and among those intoxicated by Roundup [15], the most used herbicide in the world, at least 1 abnormal ECG was detected in $80 \%$ of patients and both QTC prolongation and arrhythmias along with first degree atrio-ventricular block were observed. However, the actual mechanistic effects were not explained as, unbelievably, no investigations were performed in cardiac mammalian tissues principally based on the idea that $\mathrm{GBH}$ or Roundup are inert components when animals or humans are exposed acutely [15]. It was 
quite rational therefore to investigate in vitro rat and rabbit ventricular tissues by standard electrophysiology to assess, for the first time, whether $A D_{90}$ increase (the in vitro counterpart of QTC prolongation) might follow to short superfusion of Roundup, which was seen in conjunction with a high incidence of severe arrhythmias and of conduction blocks at the highest concentrations [16]: Figure 2 illustrates the rabbit case. Arrhythmias and conduction blocks may follow to significant $I_{\mathrm{Na}}$ and $I_{\mathrm{K}}$ blocking properties [16], possibly related to the cation chelator ability [17] of glyphosate and to decreased ${ }^{45} \mathrm{Ca}^{++}$influx through L-type voltage-dependent $\mathrm{Ca}^{++}$-channels shown by both Roundup and glyphosate [18].

Although it will be critical to further study the various components of $I_{K}$ to assess which contributes the most, the translational aspects here were nicely shown and human risks after acute intoxication [14] were linked to animal ones [16] demonstrated by electrophysiological changes seen in mammalian ventricular myocardium, including conduction blocks and arrhythmias among GBH-mediated effects. The importance of the experimental investigations to elucidate the intoxication mechanisms by Roundup (and/or GBH in general) in humans is indeed very

Figure 2. Representative example of serious premature ventricular contractions occurring in rabbit ventricular tissues in vitro after Roundup superfusion. As compared to baseline experiment, frequent premature ventricular beats were seen after 30 min of Roundup 50 ppm superfusion (modified from [15]). Also note $\mathrm{APD}_{90}$ increase that in 6 experiments, from $131 \pm 2$ (mean $\pm \mathrm{SE}$ ) ms reached $153 \pm 9$ ms $(p<0.005)[16]$.
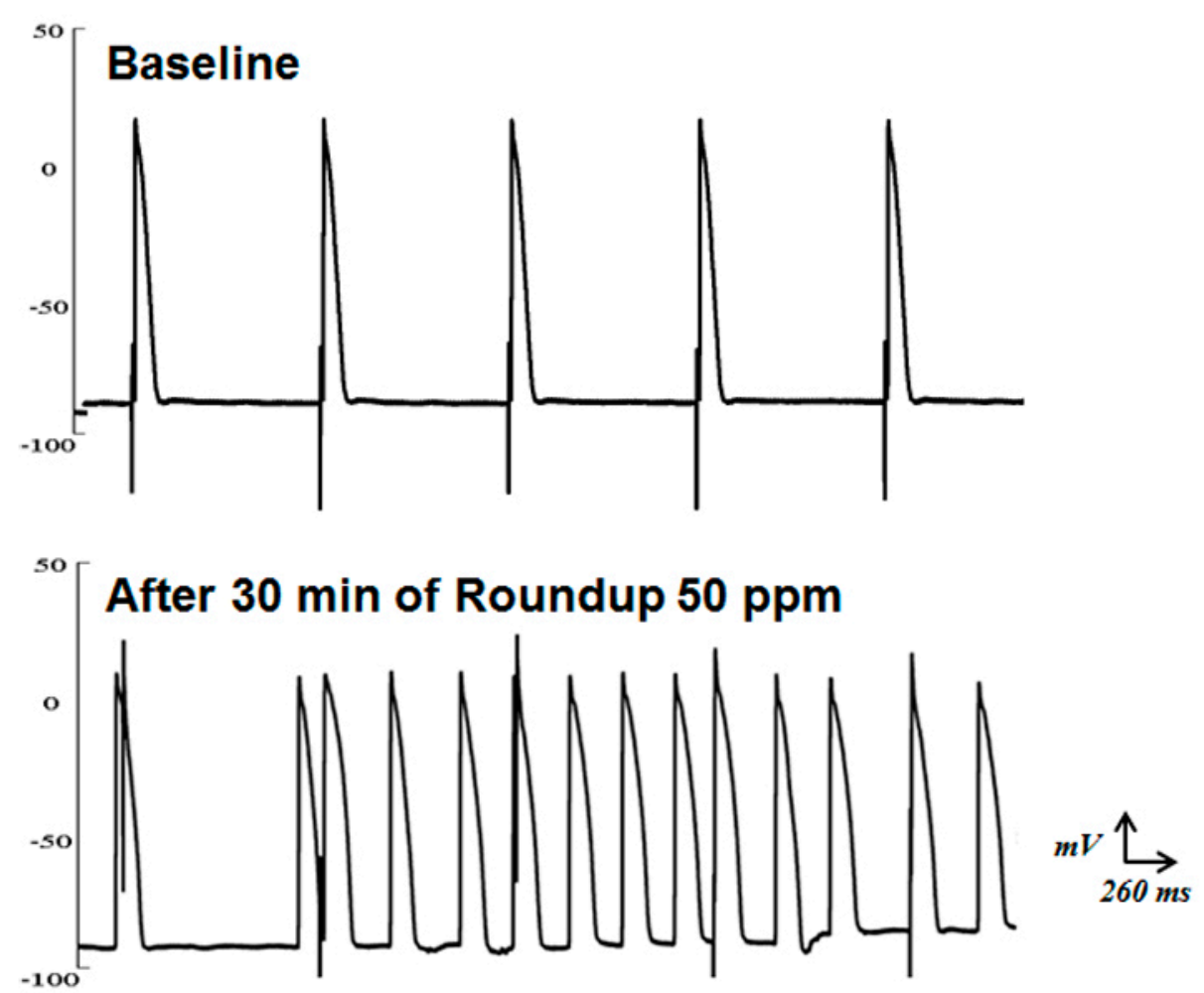
high, since a large proportion among as many as 25 million agricultural workers in the developing world suffers an episode of herbicide poisoning every year [16]. This research deserves top priority, ideally from independent Investigators, in order to confirm the findings and further define the role of $\mathrm{I}_{\mathrm{K}}$. Furthermore, it represents an example of how clinically formulated questions may be addressed in the laboratory to develop a translational impact on future treatment.

\section{Translational research in Cardiology: from the bench to the bedside}

Two definite clinically-driven examples of basic research are presented: firstly the research that has been performed around the use of left ventricular assist devices (LVADs) for the treatment of heart failure and that has exploded into new hypotheses and different directions of clinical research through a journey into basic research, and secondly the discovery of the pace-maker current $I_{f}$ and its inhibitor ivabradine and the subsequent highs and lows in the clinical applications. The very debated, expensive, and so far yet to be proven effective, use of stem cells for regenerative purposes, is deliberately omitted from this analysis, as this deserves a separate and more detailed account that will be covered extensively in future issues of the Journal.

LVADs have been developed in the last half century in the quest for replacement of the injured heart with an artificial heart [19]. LVADs are mechanical pumps that are surgically implanted and can support the circulation when cardiac function is insufficient, while unloading the LV. Originally extracorporeal and short-term, they can now be implanted in the chest for long periods of time (months/years) and successfully used in patients with severe heart failure as a bridge-to-transplantation and even as permanent treatment (destination therapy) [19]. Despite significant technical ad- vances, LVAD use is significantly hindered by a very high incidence of serious complications and cost, and can affect quality of life, thus remaining as a palliative, not a curative option for most patients [20]. In early cases when explantation of the devices was required due to technical faults or complications, it was noticed that, after LVAD treatment, there was an obvious reduction in heart size and an improvement in myocardial performance [21]; this suggested the possibility that the dysfunctional spiral that leads to permanent remodeling and the clinical syndrome of heart failure can be reversed by unloading. This observation triggered a flurry of basic research to describe details and understand mechanisms, in a typical translational example, with the goal to feed back to the clinic and optimize this modality for heart failure treatment ("bridge-torecovery") $[22,23]$. Two major platforms of "basic" research were developed. The first employs myocardial biopsies from patients, leveraging from the unique set of circumstances where relatively large amounts of myocardium can be ethically obtained at two time points from the same LVAD patient: the first - LVAD core - at the time of the implantation of the device in the LV and the second at the time of LVAD explantation during cardiac transplantation. Moreover, to overcome problems due to small patient numbers, high variability and lack of normal myocardium in human studies, a number of animal models of chronic mechanical unloading and heart failure were developed [24]. A detailed account of these studies is outside the scope of this Editorial and can be found elsewhere [22,25] but the results were extraordinary: a large number of parameters tested, from the hypertrophic response to beta adrenergic signaling, from gene expression to metabolic pathways, were found to be normalized or significantly improved after unloading (reverse remodeling). This demonstrated that the pathological substrate of heart failure, previously considered a set of irreversible degenerative changes, can in fact be reversed and that recovery from 
heart failure is possible. The interaction between basic and clinical studies in this field did not stop there: the lack of a direct correlation between reverse alterations of the substrate and clinical recovery following LVAD treatment [26] (the latter remains low, with only $5-10 \%$ devices explanted) initiated another step in the translational journey to establish a causal relationship. That is, specific biological mechanisms linked to clinical recovery or linked to its failure were needed. One of these studies looked at a population of patients treated with a combination of VAD and pharmacological therapy and linked to a higher recovery success rate (Figure 3) [27]. It was found that an improvement in sarcoplasmic reticulum (SR) calcium cycling of ventricular cardiomyocytes is associated with clinical recovery, while the reduction in cell size following LVAD was not important. This is clinically relevant and translational for two reasons: the first is that SR calcium regulation becomes an essential

Figure 3. Ventricular biopsies where taken at the time of LVAD implantation in patient with severe heart failure (HF - pre LVAD). Following treatment, some patients showed a marked regression of clinical HF parameters and the LVAD could be explanted without recurring to cardiac transplantation. During surgery, ventricular samples were collected (clinical recovery- post LVAD). In other patients LVAD treatment did not result in clinical improvement and the patients underwent cardiac transplantation during which ventricular samples were collected (HF - post LVAD). Myocytes were isolated from the 3 groups of biopsies and sarcoplasmic reticular (SR) calcium content was measured using microelectrode techniques. ${ }^{*}=p<0.05 ;{ }^{*}=p<0.01$; mito $=$ mitochondrial). Modified from [27].

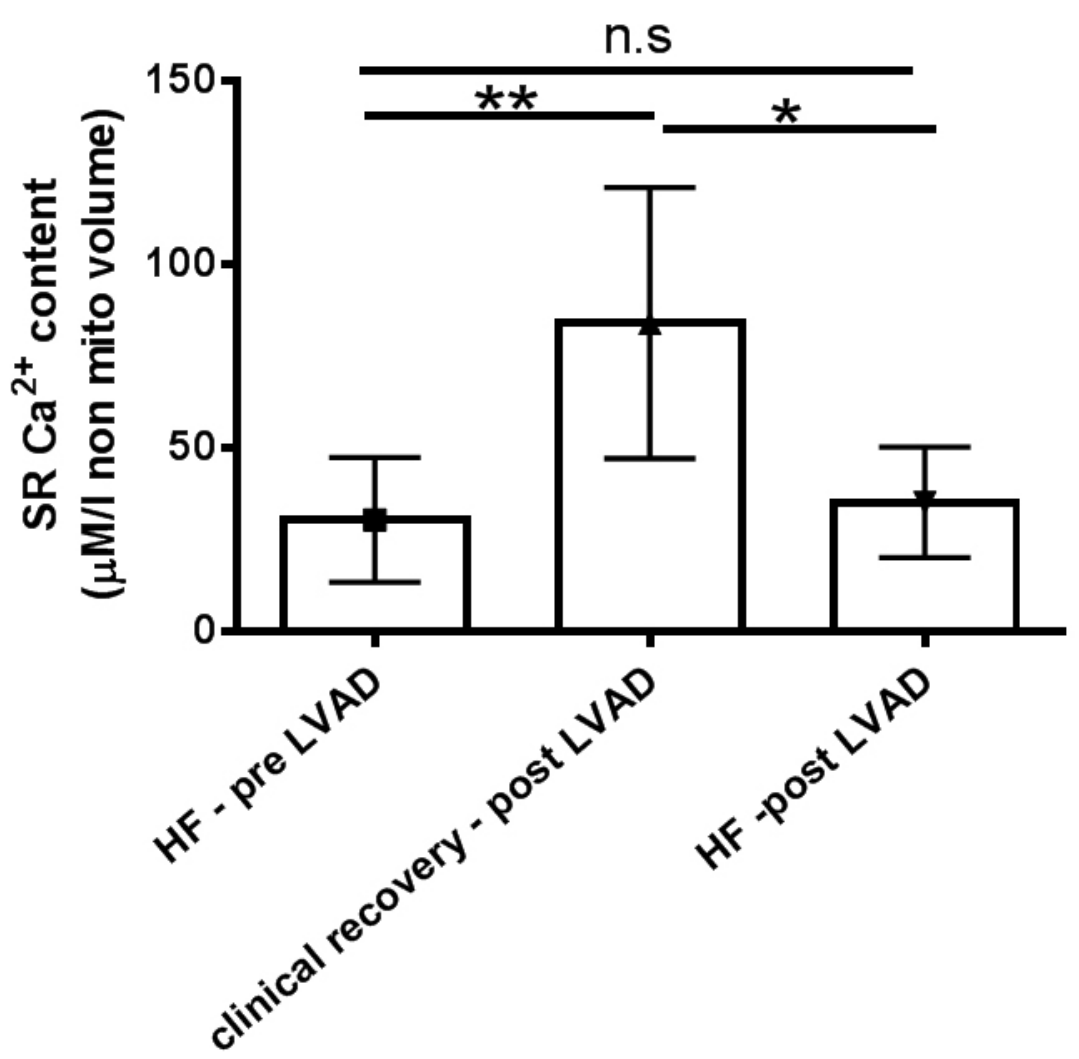


therapeutic target for recovery from heart failure; these data have supported novel and exciting clinical approaches aimed to target the SR directly (e.g. the gene therapy trials to increase the expression of SERCA in patients with heart failure [28]). The second important aspect is the discovery of an unloading-related phenomenon that we and others call "myocardial atrophy", a set of dysfunctional features that make myocyte mass and function inadequate to sustain the workload of the heart when it is reloaded $[29,30]$. Clinical strategies that limit atrophy, such as partial unloading and other "smart" unloading modalities, various pharmacological therapies and even a specific gene therapy trial to over-express SERCA in LVAD patients (clinicaltrials.gov NCT00534703) are currently being tested.

Another successful example of translational research is the fast journey from the discovery of the pace maker current $I_{f}$ in a physiology laboratory in the 1970s to recent large multi-centre clinical trials involving the $\mathrm{HCN}$ channel inhibitor ivabradine [31]. The first step of this process was to discover and link $I_{f}$ with a class of cation channels, called hyperpolarization-activated, cyclic nucleotide gated $(\mathrm{HCN})$ channels and which present predominantly in the SA node [32]. Subsequently, the relationship of $\mathrm{HCN}$ function with beta adrenergic regulation was described, but all this was still within the domain of basic physiology research. The translational part began when it was shown that pharmacological inhibition of these channels reduces heart rate and it was hypothesized that this can be exploited clinically to reduce metabolic stress in the injured myocardium (the clinical starting point). Unlike beta adrenergic blockade, this approach does not hinder myocardial contractility and has limited effects outside a pure heart rate reducing (HRR) action [32]. Defining these properties and their clinical applications has been a constructive translational exercise which has led to the development of the HCN inhibitor ivabradine followed by the BEAUTIFUL trial in more than 10,000 patients with ischemic heart disease and LV systolic dysfunction[33] and the SHIfT trial in more than 6,000 heart failure patients [34]. Both trials showed that the rate of hospitalization is significantly reduced in patients with basal heart rate $>70$ bpm although overall mortality is not affected by this treatment. A recent clinical trial in $>19,000$ patients with stable coronary disease but without clinical heart failure (SIGNIFY) confirmed the observation that mortality is not affected by the addition of ivabradine to standard treatment, casting a shadow on its future use in clinical practice [35]. Overall, patients with LV dysfunction seem to benefit from treatment with ivabradine, possibly through an effect on LV remodeling [36]. This has triggered a series of basic research studies in two major areas: the first is the possibility that ivabradine exerts a beneficial effect in patients with heart failure where HCN channels are expressed in ventricular myocytes and can contribute to the arrhythmogenic substrate [37]. The other is the occurrence of HRR-independent effects, such as a reduction in myocardial fibrosis demonstrated in animal models of heart failure and that open a new clinical application for this target (Figure 4) [38]. The latter observation feeds directly into clinical applications where anti-fibrotic therapy is taking central stage and now requires clinical validation and testing. 
Figure 4. Myocardial fibrosis, quantified using picrosirius red staining and expressed as collagen area fraction, was measured in fixed sections of rat hearts with established heart failure (HF) 16 weeks after coronary artery ligation. One group of animals was treated with ivabradine (IVA) for 4 weeks. Another group was treated with metoprolol for 4 weeks, to induce a similar $10-15 \%$ heart rate reduction (HRR). Sham-operated animals were used as control. Ivabradine induced a HRR-independent reduction of myocardial fibrosis. ${ }^{* * *}=p<0.001$. Modified from [38].

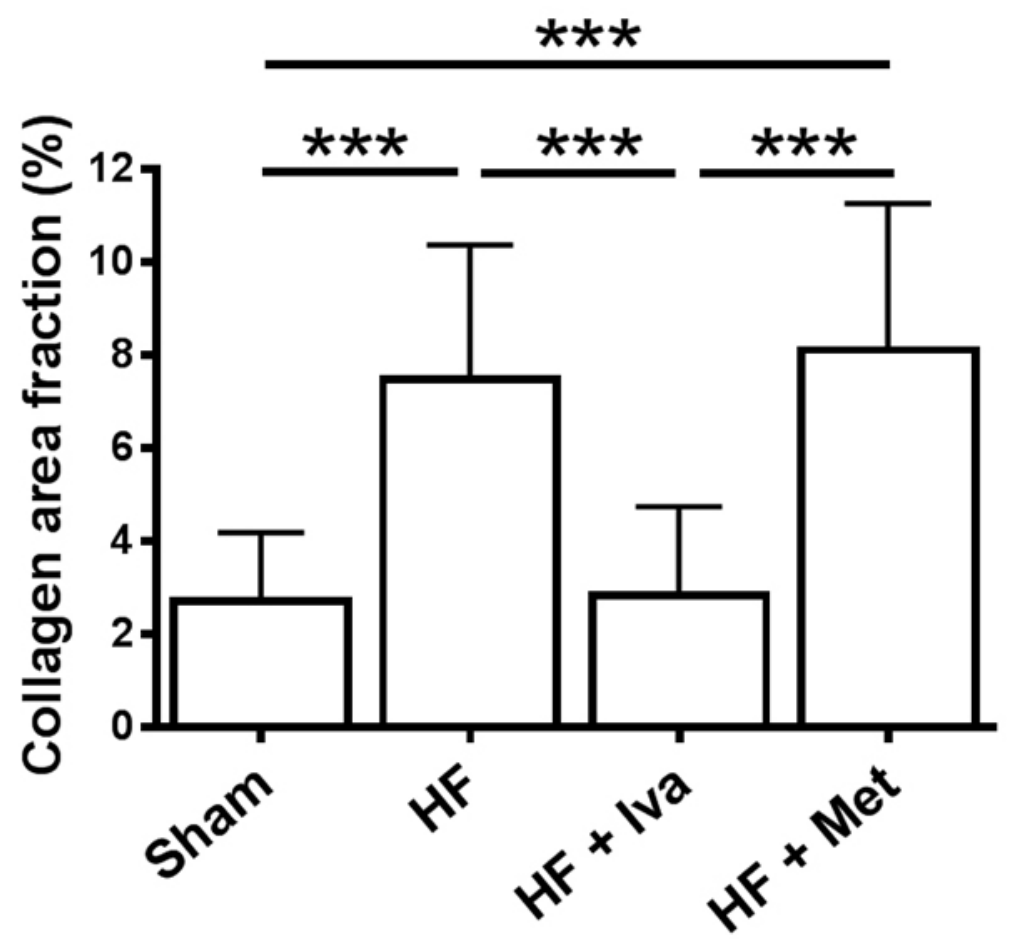

\section{Translational research in general is not a Phoenix}

The examples reported here show how much overlap exists between basic, clinical and translational research in Cardiology, with even the direction of applicability (bedside-to-bench or bench-to-bedside) representing a matter of debate. The notion of an intricate relationship between clinical and fundamental aspects requires that limits and targets of translational research are defined. Two important quantitative parameters should guide us. The first is time: there should be a tight temporal connection between basic and clinical studies as part of a unitary effort; the two aspects should swiftly follow each other, possibly even be performed as a contemporary exercise. The second quantitative aspect is the impact of basic and clinical research on each other: there should be a real and significant clinical applicability following basic observations, which affects every-day clinical practice and opens new therapeutic targets. Vice versa clinical studies should open new fields of fundamental research that would not be otherwise performed and that significantly change the way we understand mechanisms. Without a strong component in these two parameters, time and impact, the concept of translational research remains vague and its existence may even be questioned. 
It was January 26, 1790 in Vienna that Mozart's K 588 (Cosi fan tutte, ossia La scuola degli amanti: an Italian-language opera buffa in two acts) was first performed. The libretto was written by Lorenzo Da Ponte who stated "È la fede delle femmine come l'Araba fenice: che vi sia ciascun lo dice, dove sia nessun lo sa!" He literally derived it from Pietro
Metastasio, considered the most important writer of opera seria libretti, who created the famous dicto "Come I'araba Fenice, che vi sia ciascun lo dice, dove sia nessun lo sa" (from Demetrio, act II, scene III) meant to describe something uncomparable, impossibile to find, unique and/or difficult to catch (Figure 5).

Figure 5. Greek mythology reports the Phoenix as a 500 to 1400-year living bird that is cyclically regenerated or reborn. His motto (post fata resurgo) well depicts the situation. He typically dies by fire and according to several classical Authors could symbolize renewal in general as well as the sun, time, the empire, metemspychosis, consecration, resurrection, life in the heavenly Paradise, Christ, Mary, virginity, the exceptional man, and certain aspects of Christian life. It is pictured in medieval art as endowed with a nimbus, which emphasizes the bird's connection with the sun. There was no consensus about its coloration: some thought that the bird had a peacocklike coloring and Herodotus' claim of red and yellow is popular in many versions of the story on record. In terms of size the Phoenix was described similar to an eagle but others declared it was larger. A variety of cultures presents with analogues to the Phoenix: the Arabic Anga, the Hindu Garuda and Gandaberunda, the Russian Firebird, the Persian Simorgh, the Turkish Zümrüdú Anka, the Tibetan Me byi karmo, the Chinese Fenghuang and the Japanese $\mathrm{H}$-ōo.

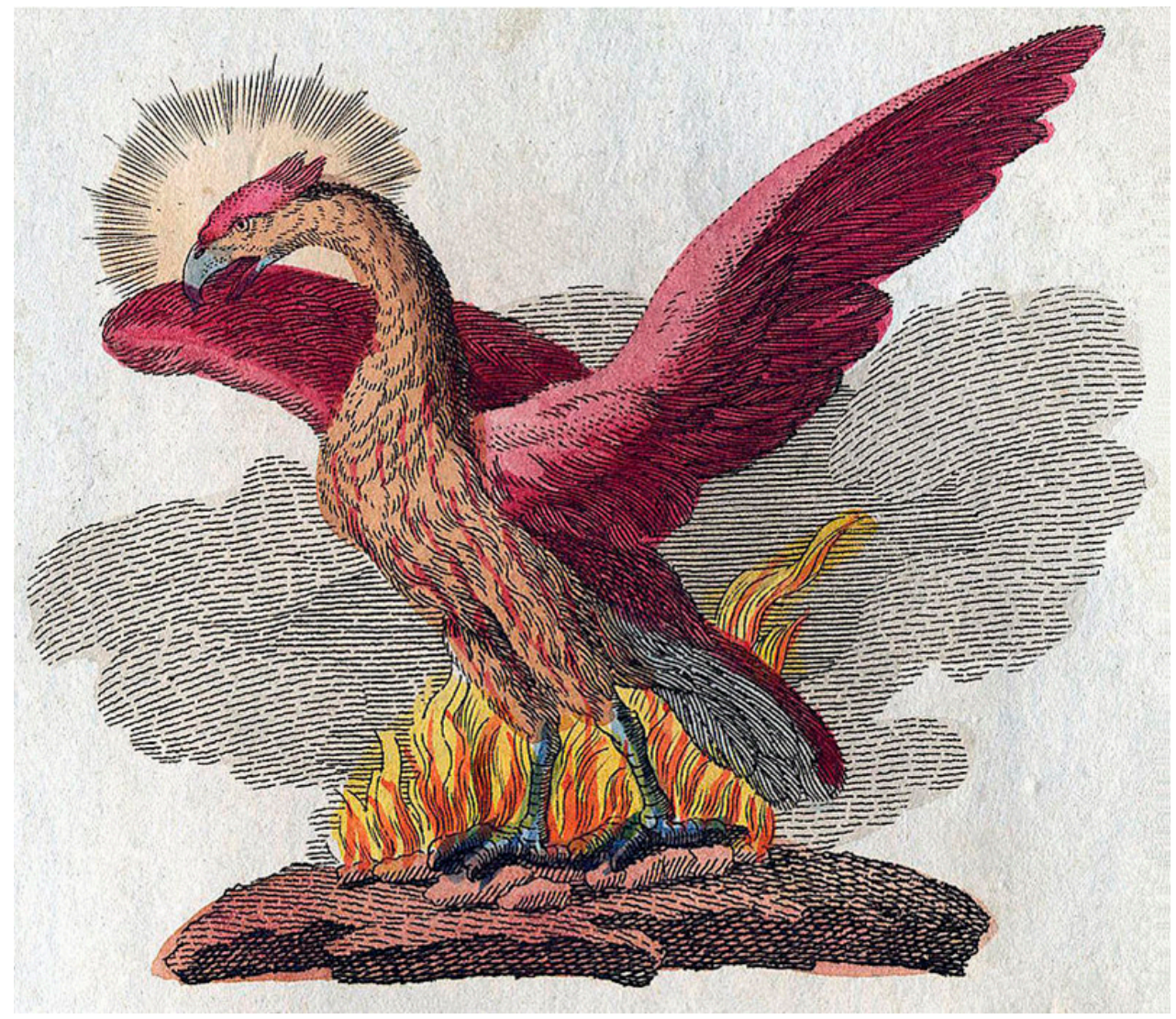


Translational research in Cardiology should not be a Phoenix. As cardiovascular factors are proportionately the most important ones and are heavily involved in overall survival [39], it is essential that clinical questions formulated to solve a given problem or to add to the understanding of pathophysiological concepts or mechanisms for prevention, diagnosis or treatment are of impact and swiftly follow in time. In particular there should be a measurable impact and it should be shown that this adds as compared to previous factors or knowledge. For example ECG voltage criteria of LV hypertrophy $(\mathrm{LVH})$ were shown to contribute to the prediction of sudden death in a residential cohort [40]. However, in a large cohort of black and white participants (all-comers) from the ARIC Study, they were associated with cardiovascular disease or coronary (CVD/CHD) risk but did not significantly improve CVD and CHD events risk prediction beyond the most used Framingham risk equations [41]. Therefore, the impact of ECG-LVH indexes might be comparatively minor or adding few to easier to obtain or less expensive risk factors. The long time needed $[39,40]$ to reach these conclusions may nicely show the difficulties of translational research when passing from a basic discipline such as epidemiology [5] to the clinical application in an allcomers context. On the other hand, the promise of imaging modalities to unveil pathophysiological problems of LV function [2,7-10] might enable the detection of subtle abnormalities well in advance as compared to the natural evolution not driven by these much effective translational methods [42]. The missing thread could be that changes in LV geometry (concentric versus eccentric hypertrophy) are more important, for prognostic significance or predicting purposes $[5,43]$, than ECG-LVH indexes $[39,40]$ and thus specific imaging modalities [42] should be performed to better index anatomical correlates. Finally, LV function itself and its many receptor-, channels- and molecular-mediated interferences $[22-31,38]$ will probably remain the Golden
Fleece to look actively for, in order to detect with the highest probability an impacting significance in Translational Cardiology.

It is what we expect at the International Archives of Medicine. We hope to have on board of a novel Argo boat a series of Heroes who will strive to connect basic and clinical Sciences bidirectionally, offering the evidence in an understandable way and transmitting to the Clinicians the essence of new impacting knowledge in plane terms and a timely fashion.

\section{References}

1. "Translational Research" Merriam-Webster.com. MerriamWebster, Available at: http://www.merriam-webster.com/ dictionary/translationalresearch. Accessed December 15, 2014.

2. Martínez-Legazpi P, Bermejo J, Benito $Y$, Yotti R, Pérez Del Villar C, González-Mansilla A, Barrio A, Villacorta E, Sánchez PL, Fernández-Avilés F, del Álamo JC. Contribution of the diastolic vortex ring to left ventricular filling. J Am Coll Cardiol 2014; 64: 1711-21.

3. Landis S. Back to basics: a call for fundamental neuroscience research. Available at: http://blog.ninds.nih.gov/. Accessed December 15, 2014.

4. Editorial. EBioMedicine: Bridging two cultures to improve health. EBioMedicine 2014; 1: 1. http://dx.doi.org/10.1016/j. ebiom.2014.10.007

5. Lauer MS. The basic Science that is Epidemiology. J Am Coll Cardiol Cardiovasc Imag 2014; 7: 879-81.

6. Glenn C, Goldthwaite MA. The St. Martin's Guide to Teaching Writing. Boston, MA: Bedford/St. Martin's. 2008, pp.151-156.

7. Piras P, Evangelista A, Gabriele S, Nardinocchi P, Teresi L, Torromeo C, Schiariti M, Varano V, Puddu PE. 4D-analysis of left ventricular cycle using Procrustes motion analysis. Plos One 2014; 9(1): e86896. The PLOS ONE Staff. Correction: 4D-Analysis of Left Ventricular Heart Cycle Using Procrustes Motion Analysis. PLos One 2014; 9: e94673.

8. Evangelista A, Gabriele S, Nardinocchi P, Piras P, Puddu PE, Teresi L, Torromeo C, Varano $\mathrm{V}$. On the strain-line pattern in the real human left ventricle. J Biomechanics 2014, http://dx.doi. org/10.1016/j.jbiomech.2014.12.028

9. Madeo A, Piras $P$, Evangelista A, Giura G, Dominaci $T$, Nardinocchi P, Varano V, Chialastri C, Puddu PE, Torromeo C. Unveiling hypertrophic cardiomiopathy mechanics by means of left ventricular morphological trajectories in 4D. Eur Heart J Cardiovasc Imaging 2014; 15(suppl 2): ii196-ii223.

10. Madeo A, Piras P, Re F, Gabriele S, Nardinocchi P, Teresi L, Torromeo C, Chialastri C, Schiariti M, Giura G, Evangelista A, 
Dominici T, Varano V, Zachara E, Puddu PE. A new 4D trajectorybased approach unveils abnormal LV revolution dynamics in hypertrophic cardiomyopathy. Plos One 2014, submitted

11. Puddu PE, Jouve R, Torresani J, Sambuc R, Jouve A. Prolonged electrical systole in acute myocardial infarction. J Electrocardiol 1980; 13: 337-340.

12. Puddu PE, Jouve R, Torresani J, Jouve A. QT interval and primary ventricular fibrillation in acute myocardial infarction. Am Heart J 1981; 101: 118-120.

13. Puddu PE, Bourassa MG, Lespérance J, Hélias J, Danchin N, Goulet C. Can the mode of death be predicted in patients with angiographically documented coronary artery disease? Clin Cardiol 1983; 6: 384-395.

14. Kim YH, Lee JH, Hong CK, Cho KW, Park YH, Kim YW, Hwang SY. Heart rate-corrected QT interval predicts mortality in glyphosate-surfactant herbicide-poisoned patients. Am J Emerg Med 2014; 32: 203-207.

15. Gress S, Lemoine S, Séralini GE, Puddu PE. Glyphosate-based herbicides potently affect cardiovascular system in mammals: review of the literature. Cardiovasc Toxicol 2014 doi: 10.1007/ s12012-014-9282-y.

16. Gress S, Lemoine S, Puddu PE, Séralini GE, Rouet R. Mechanisms involved in the cardiotoxic electrophysiological effects of the herbicide Roundup $₫$ in rat and rabbit ventricular myocardium in vitro. Cardiovasc Toxicol 2014 doi: 10.1007/s12012-0149299-2.

17. Olorunsogo OO. Modification of the transport of protons and $\mathrm{Ca}^{2+}$ ions across mitochondrial coupling membrane by N-(phosphonomethyl)glycine. Toxicology 1990; 61: 205-209.

18. De Liz Oliveira Cavalli VL, Cattani D, Heinz Rieg CE, Pierozan P, Zanatta L, Benedetti Parisotto E, Zamoner A. Roundup disrupts male reproductive functions by triggering calcium-mediated cell death in rat testis and Sertoli cells. Free Rad Biol Med 2013; 65 : 335-346.

19. Terracciano CM, Miller LW, Yacoub MH. Contemporary use of ventricular assist devices. Annu Rev Med 2010; 61: 255-270.

20. Wever-Pinzon O, Drakos SG, Kfoury AG, Nativi JN, Gilbert EM, Everitt M, Alharethi R, Brunisholz K, Bader FM, Li DY, Selzman $\mathrm{CH}$, Stehlik J. Morbidity and mortality in heart transplant candidates supported with mechanical circulatory support: is reappraisal of the current United network for organ sharing thoracic organ allocation policy justified? Circulation 2013; 127 : 452-462.

21. Frazier $\mathrm{OH}$, Benedict $C R$, Radovancevic $B$, Bick RJ, Capek $P$, Springer WE, Macris MP, Delgado R, Buja LM. Improved left ventricular function after chronic left ventricular unloading. Ann Thorac Surg 1996; 62: 675-681.

22. Soppa GK, Barton PJ, Terracciano CM, Yacoub MH. Left ventricular assist device-induced molecular changes in the failing myocardium. Curr Opin Cardiol 2008; 23: 206-218.

23. Ibrahim M, Terracciano CM, Yacoub $\mathrm{MH}$. Bridge to recovery: what remains to be discovered? Cardiol Clin 2011; 29: 531-547.
24. Ibrahim M, Navaratnarajah M, Kukadia P, Rao C, Siedlecka U, Cartledge JE, Soppa GK, Van DC, Yacoub MH, Terracciano CM. Heterotopic abdominal heart transplantation in rats for functional studies of ventricular unloading. J Surg Res 2013; 179: e31-39.

25. Birks EJ. Molecular changes after left ventricular assist device support for heart failure. Circ Res 2013; 113: 777-791.

26. Drakos SG, Kfoury AG, Stehlik J, Selzman CH, Reid BB, Terrovitis JV, Nanas JN, Li DY. Bridge to recovery: understanding the disconnect between clinical and biological outcomes. Circulation 2012; 126: 230-241.

27.Terracciano CM, Hardy JP, Birks EJ, Khaghani A, Banner NR, Yacoub $\mathrm{MH}$. Clinical recovery from end-stage heart failure using left-ventricular assist device and pharmacological therapy correlates with increased sarcoplasmic reticulum calcium content but not with regression of cellular hypertrophy. Circulation 2004; 109: 2263-2265.

28. Jessup M, Greenberg B, Mancini D, Cappola T, Pauly DF, Jaski B, Yaroshinsky A, Zsebo KM, Dittrich H, Hajjar RJ. Calcium upregulation by percutaneous administration of gene therapy in cardiac disease (CUPID): a phase 2 trial of intracoronary gene therapy of sarcoplasmic reticulum $\mathrm{Ca}^{2+}$-ATPase in patients with advanced heart failure. Circulation 2011; 124: 304-313.

29. Ibrahim M, Al MA, Navaratnarajah M, Siedlecka U, Soppa GK, Moshkov A, bou Al-Saud S, Gorelik J, Yacoub MH, Terracciano $\mathrm{CM}$. Prolonged mechanical unloading affects cardiomyocyte excitation-contraction coupling, transverse-tubule structure, and the cell surface. FASEB J 2010; 24: 3321-3329.

30. Ibrahim M, Terracciano CM. Reversibility of T-tubule remodelling in heart failure: mechanical load as a dynamic regulator of the T-tubules. Cardiovasc Res 2013; 98: 225-232.

31. Terracciano CM, Yacoub MH. Heart failure: A SHIFT from ion channels to clinical practice. Nat Rev Cardiol 2010; 7: 669-670.

32. Difrancesco D. The role of the funny current in pacemaker activity. Circ Res 2010; 106: 434-446.

33. Fox K, Ford I, Steg PG, Tendera M, Ferrari R. Ivabradine for patients with stable coronary artery disease and left-ventricular systolic dysfunction (BEAUTIFUL): a randomised, double-blind, placebo-controlled trial. Lancet 2008; 372: 807-816.

34. Swedberg K, Komajda M, Bohm M, Borer JS, Ford I, DubostBrama A, Lerebours G, Tavazzi L. Ivabradine and outcomes in chronic heart failure (SHIFT): a randomised placebo-controlled study. Lancet 2010; 376: 875-885.

35. Fox K, Ford I, Steg PG, Tardif JC, Tendera M, Ferrari R. Ivabradine in stable coronary artery disease without clinical heart failure. N Engl J Med 2014; 371: 1091-1099.

36. Tardif JC, O'Meara E, Komajda M, Bohm M, Borer JS, Ford I, Tavazzi L, Swedberg K. Effects of selective heart rate reduction with ivabradine on left ventricular remodelling and function: results from the SHIFT echocardiography substudy. Eur Heart J 2011; 32: 2507-2515. 
37.Cerbai E, Barbieri M, Mugelli A. Characterization of the hyperpolarization-activated current, I(f), in ventricular myocytes isolated from hypertensive rats. J Physiol 1994; 481 (Pt 3): 585591.

38. Navaratnarajah $M$, Ibrahim $M$, Siedlecka $U$, Van DC, Shah $A$, Gandhi A, Dias P, Sarathchandra P, Yacoub MH, Terracciano CM. Influence of ivabradine on reverse remodelling during mechanical unloading. Cardiovasc Res 2013; 97: 230-239.

39. Puddu PE, Menotti A, Tolonen H, Nedeljkovic S, Kafatos A. Determinants of 40-year all-cause mortality in the European cohorts of the Seven Countries Study. Eur J Epidemiol 2011; 26: 595-608.

40. Lanti M, Puddu PE, Menotti A. Voltage criteria of left ventricular hypertrophy in sudden and nonsudden coronary artery disease mortality: the italian section of the Seven Countries Study. Am J Cardiol 1990; 66: 1181-1185.

41. Okwuosa TM, Soliman EZ, Lopez F, Williams KA, Alonso A, Ferdinand KC. Left ventricular hypertrophy and cardiovascular disease risk prediction and reclassification in blacks and whites: The Atherosclerosis Risk in Communities Study. Am Heart J 2015; 169: 155-161.

42. Lamata P, Casero R, Carapella V, Niederer SA, Bishop MJ, Schneider JE, Kohl P, Grau V. Images as drivers of progress in cardiac computational modelling. Progr Biophys Mol Biol 2014; 115; 198-212.

43. Lieb W, Gona P, Larson MG, Aragam J, Zile MR, Cheng S, Benjamin EJ, Vasan RS. The natural history of left ventricular geometry in the community. Clinical correlates and prognostic significance of change in LV geometric pattern. J Am Coll Cardiol Img 2014; 7: 870-878.

\section{Comment on this article:}

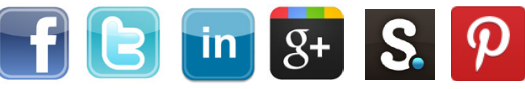

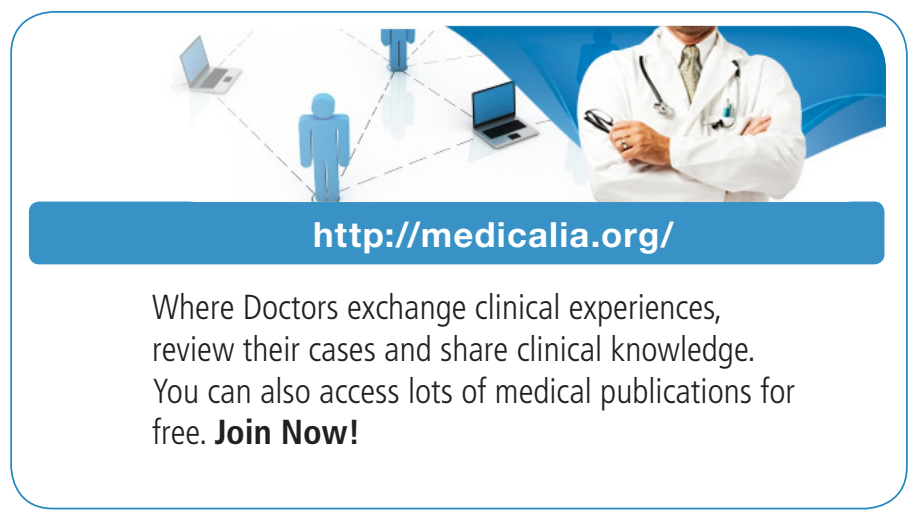

\section{Publish with iMedPub}

\section{http://www.imed.pub}

International Archives of Medicine is an open access journal publishing articles encompassing all aspects of medical science and clinical practice. IAM is considered a megajournal with independent sections on all areas of medicine. IAM is a really international journal with authors and board members from all around the world. The journal is widely indexed and classified Q1 in category Medicine. 